\title{
Effects of injection Shenkang and alprostadil combination on contrast-induced nephropathy in patient with diabetes complicated with mild to moderate renal insufficiency
}

\author{
Yanfang Zhang1, Jianxun Sun', Hui Liu² and Xiaolin Zhai ${ }^{2}$ \\ ${ }^{1}$ Luoyang Vocational and Technical College, 471000, Luoyang, Henan, China; ${ }^{2}$ Luoyang Central Hospital, 471000, \\ Luoyang, Henan, China.
}

\begin{tabular}{|c|c|}
\hline \multicolumn{2}{|l|}{ Article Info } \\
\hline Received: & 4 April 2017 \\
\hline Accepted: & 29 July 2017 \\
\hline Available Online: & 25 August 2017 \\
\hline \multicolumn{2}{|c|}{ DOI: $10.3329 /$ bjp.v12i3.32070 } \\
\hline \multicolumn{2}{|c|}{$\begin{array}{l}\text { Cite this article: } \\
\text { Zhang Y, Sun J, Liu H, Zhai X. Effects } \\
\text { of injection Shenkang and alprostadil } \\
\text { combination on contrast-induced } \\
\text { nephropathy in patient with diabetes } \\
\text { complicated with mild to moderate } \\
\text { renal insufficiency. Bangladesh J } \\
\text { Pharmacol. 2017; } 12: 308-12 \text {. }\end{array}$} \\
\hline
\end{tabular}

\begin{abstract}
The diabetic patients $(n=109)$ with mild or moderate renal insufficiency underwent coronary intervention were randomly divided into control group and observation group. The patients in observation group were treated with injection Shenkang and alprostadil before operation. The results showed that the level of serum creatinine, creatinine clearance and blood urea nitrogen had no significant difference $(\mathrm{p}>0.05)$ before and postoperative day 1 and day 2 in both groups. However, the value of serum creatinine, creatinine clearance and blood urea nitrogen were significantly different on post-operative day 3 $(p<0.05)$. The incidence of contrast-induced nephropathy was $14.8 \%$ in control group, and markedly higher than observation group $(3.6 \%) \quad(p<0.05)$. Shenkang injection combined with alprostadil injection decreased the incidence of contrast-induced nephropathy in elderly patients with diabetes mellitus complicated with mild to moderate renal insufficiency.
\end{abstract}

\section{Introduction}

Contrast-induced nephropathy (CIN) is mainly due to the use contrast agents such as iodopa alcohol, iodixanol and beryllium sulfate in surgery in recent years. It has become the 3rd leading cause of iatrogenic acute renal failure (Feldkamp and Kribben, 2008). CIN resulted in acute or transient renal function damage, even permanent damage or renal failure. Numerous studies suggest that the incidence rate of $\mathrm{CIN}$ is lower than $2 \%$ in the general population. However, the incidence rate of CIN is $20 \sim 50 \%$ in patients with metabolic syndrome (such as diabetes, coronary heart disease, renal insufficiency), even up to $90 \%$ ( $\mathrm{Li}$ et al., 2010). Meanwhile, patients with diabetes and renal insufficiency have a significantly increased risk of CIN (Isobe et al., 2013). Although prevention measures are improved, the increasing incidence of CIN is still challenging ( $\mathrm{Lu}$ et al., 2017). Therefore, investigating possible prevention and treatment strategies for CIN is attracting increased interest in medical science.
Shenkang injection had a positive effect in the prevention of CIN, which used for chronic renal insufficiency (Wang et al., 2013). Alprostadil, an active ingredient of prostate E1 (PEG-E1), plays a protective role in renal function. Moreover, it markedly relax the blood vessels, inhibit platelet aggregation, stimulate the intestinal and uterine smooth muscle (Miao et al., 2013; Li et al., 2012). However, Shenkang injection combined with alprostadil injection on the prevention effect of CIN is still needed more clinical experimental studies to confirm.

This study investigated Shenkang injection combined with alprostadil injection on CIN in elderly patients with diabetes mellitus complicated with mild to moderate renal insufficiency.

\section{Materials and Methods}

The study included 109 cases of diabetes with mild to moderate renal dysfunction in elderly patients admitted 
during the period November 2013 to November 2015. Diagnostic criteria of Western medicine were a) patient complaints of more than 6-10 years, b) the rate of microalbumin excretion was twice as high as $30 \mathrm{mg} / 24$ hours, or c) significant proteinuria (the rate of microalbumin excretion higher than $0.5 \mathrm{~g} / 24$ hours); d) hypertension, e) azotemia and so on. In addition, random blood glucose concentration higher than 11.1 $\mathrm{mmol} / \mathrm{L}$ after oral glucose tolerance test, or PBG higher than $11.1 \mathrm{mmol} / \mathrm{L}$, or FPG higher than $7.0 \mathrm{mmol} / \mathrm{L}$. Diagnostic criteria of Chinese medicine (according to the Chinese Society of Chinese Medicine Symposium on the fifth diagnostic criteria) were: a) consumptive thirst involving kidney, b) kidney blood deficiency syndrome, c) wet muddy intrinsic stasis, d) soreness, e) weakness of waist and knees, f) lack of energy, g) weight loss, h) lower extremity edema, i) poor appetite, j) stool unhappy, k) facial skin dull, l) tongue or dark purple color weakness points and $\mathrm{m}$ ) itchy skin.

Excluding other causes of renal dysfunction, we compared the levels of serum creatinine before and after contrast agent used. Serum creatinine levels were increased by $0.5 \mathrm{mg} / \mathrm{dL}$ or $44 \mu \mathrm{mol} / \mathrm{L}$, or a relative increase higher than $25 \%$ within three days after used contrast agent, then diagnosed as CIN. Inclusion criteria were: a) Elder than 63 years of age, meet the above criteria; b) Intends percutaneous coronary (including coronary angiography, coronary balloon angioplasty, coronary stent implantation); c) Mild to moderate renal insufficiency, and nearly 7 days without infusion or injection of alprostadil injection; d) Medical records complete, willing to accept the relevant drug treatment. Exclusion criteria were: (1) Exclude congestive heart failure, aortic balloon counter pulsation, low hematocrit, hypotension, acute respiratory distress syndrome; (2) Exclude patients with severe renal insufficiency, peripheral vascular disease; (3) Incomplete condition, allergy, intolerance and not willing to patients accept the relevant drug treatment.

Patients were randomly divided into two groups: control group $(n=54)$ and observation group $(n=55)$. The general information of the two group of patients were not significant (Table I). That is why, the study was comparable. The control group underwent routine hydration therapy to surgery in the preoperative $4 \sim 12$ hours to $5 \sim 24$ hours at $1.0 \mathrm{~mL} / \mathrm{kg} /$ hours speed of intravenous infusion of sodium chloride injection. The observation group was treated on the basis of hydration, one day before surgery and after 3 days of continuous use alprostadil injection $(20 \mathrm{~mL} / 10 \mu \mathrm{g}) 10$ $\mu \mathrm{g}$ and $100 \mathrm{~mL}$ sodium chloride injection, $10 \mathrm{ng} / \mathrm{kg} /$ min, intravenous infusion. At the same time, in the preoperative 3 days and 3 days after the continuous use of kidney Kang injection (20 mL/branch) 20 and 300 $\mathrm{mL} 5 \%$ glucose intravenous drip. The contrast agent used was iohexol (50 mL/branch), the two groups were $140 \mathrm{~mL}$, respectively.
Preoperative detection of clinical data was weight, gender, age and body mass index. Biochemical indicators were the levels of total cholesterol, low-density lipoprotein (LDL), high-density lipoprotein (HDL) and uric acid. Kidney function indicators were serum creatinine, creatinine clearance and blood urea nitrogen (BUN). The levels of serum creatinine, creatinine clearance and BUN were detected on postoperative day 1 to day 3. CIN was diagnosed by the level of serum creatinine which were compared preoperative renal function parameters. Biochemical indicators were provided by a automatic biochemical detector (Hitachi6700 type) using enzymatic, direct determination method for the determination of the kit by the Nanjing shenbeijia Biotechnology Co., Ltd.

The formula for the elimination of endogenous creatinine was as follows:

$=[(140$-year $) \times$ body weight $(\mathrm{kg})] /[0.818 \times$ serum creatinine (umoL/L)], where women were calculated as 0.8 .

Chinese medicine syndrome evaluation: Reference to the 1999 Chinese Medical Association of diabetes professional committee in Shandong Mingshui third meeting adopted by "Diabetes syndrome differentiation and efficacy evaluation criteria-diabetes syndrome diagnosis reference standard" (Lv et al., 1999). According to the main clinical symptoms of the patients with tongue, pulse, yin deficiency, yang deficiency type, or yin yang deficiency type diabetic nephropathy, the severity of the 34 cases was graded and given the score. Among them, no symptoms were noted as 0 point; mild symptoms as 1 point; moderate symptoms as 2 points; severe symptoms recorded as 3 points, comprehensive score was evaluated before and after treatment.

Integral ratio $=($ before treatment points - after treatment points)/before treatment point $\times 100 \%$

Finally, according to the income score ratio: Between 70 - 100\%, for the marked; between 30 - 70\% effective; $\leq 30 \%$, is invalid. Total efficiency $=$ (valid + effective $)$ cases/total number of cases $\times 100 \%$.

SPSS 17.0 data processing software was used to measure data with the mean \pm variance $(x \pm s)$ to show, in line with the normal distribution using t-test to compare the difference between the two groups. Otherwise, nonparametric test was used. The $p$ value of $<0.05$ indicated that the difference was statistically significant.

\section{Results}

As shown in Table I, there was no significant difference $(p>0.05)$ before surgery clinical data and biochemical indices in two groups. Except for weight, age, gender 
Table I

Comparison of preoperative general clinical data and biochemical indicators ( $x \pm s)$

\begin{tabular}{|c|c|c|c|}
\hline Indicators & Control group & Observation group & $\mathrm{p}$ \\
\hline Male & $\begin{array}{r}32 \\
(59.3 \%)\end{array}$ & $\begin{array}{r}31 \\
(56.4 \%)\end{array}$ & 0.763 \\
\hline Age (year) & $67.0 \pm 6.1$ & $67.5 \pm 5.5$ & 0.582 \\
\hline Body mass index $\left(\mathrm{kg} / \mathrm{m}^{2}\right)$ & $25.6 \pm 4.1$ & $25.4 \pm 3.9$ & 0.775 \\
\hline Systolic pressure ( $\mathrm{mmHg}$ ) & $132 \pm 21$ & $133 \pm 23$ & 0.816 \\
\hline Serum creatinine $(\mu \mathrm{moL} / \mathrm{L})$ & $95.1 \pm 18.1$ & $96.0 \pm 18.6$ & 0.804 \\
\hline Creatinine clearance $(\mathrm{mL} / \mathrm{min})$ & $71.2 \pm 24.4$ & $72.4 \pm 24.0$ & 0.792 \\
\hline BUN $(\mathrm{mmoL} / \mathrm{L})$ & $8.2 \pm 2.2$ & $7.7 \pm 2.0$ & 0.281 \\
\hline Total cholesterol ( $\mathrm{mmol} / \mathrm{L})$ & $4.8 \pm 1.2$ & $4.6 \pm 1.0$ & 0.467 \\
\hline LDL $(\mathrm{mmol} / \mathrm{L})$ & $2.5 \pm 0.8$ & $2.6 \pm 0.7$ & 0.506 \\
\hline HDL (mmol/L) & $1.1 \pm 0.5$ & $1.1 \pm 0.2$ & 0.604 \\
\hline BUA $(\mu \mathrm{mol} / \mathrm{L})$ & $396.6 \pm 100.8$ & $390.4 \pm 103.9$ & 0.753 \\
\hline Lesions (lesion number) & $2.2 \pm 0.9$ & $2.1 \pm 0.9$ & 0.519 \\
\hline
\end{tabular}

and other interference, the test was comparable.

Serum creatinine, creatinine clearance and BUN were detected on postoperative day 1 to day 3 . The values of serum creatinine and BUN were increased whereas the creatinine clearance values were decreased. The levels of serum creatinine, creatinine clearance and BUN were not significant difference among the two groups on postoperative day 1 and day $2(\mathrm{p}>0.05)$. However, the levels of serum creatinine, creatinine clearance and BUN in observation group were increased on postoperative day 3 . In addition, there was markedly significant difference on postoperative day 3 (Table II). It indicated that the renal function recovery in observation group was better.

The incidence of CIN in the observation group was significantly lower than that in the control group (2 cases, $3.6 \%)$ and 8 cases $(14.8 \%)$ respectively. These changes were statistically significant $\left(\chi^{2}=4.086\right.$, $\mathrm{p}<0.05)$. There was no death in the two groups during the clinical study. The total effective rates of the observation group and the control group were 25 $(46.3 \%)$ and $39(70.9 \%)(p<0.05)$, respectively.

\section{Discussion}

CIN is a major sequelae associated with the use of contrast agents in recent years. Although the incidence is low in general patients, the incidence in metabolic syndromes such as coronary heart disease, diabetes, kidney disease and other special populations, especially some high-risk patients was significantly increased, up to $50 \%$ (Parfrey et al., 1989; Gleeson et al., 2004). Due to the reduction of renal units in diabetes and renal insufficiency, it resulted in a variety of shrinking vascular factor release disorders. Moreover, it caused long-term renal vasoconstriction, prolonged renal tubular exposure, and injury or even death. At the same time, it caused medulla hypoxia and resulted in oxidative stress, and further accelerated the death of renal tubular cells (Kato et al., 2008; Menting et al.,

\begin{tabular}{|c|c|c|c|c|}
\hline \multicolumn{5}{|c|}{ Table II } \\
\hline \multicolumn{5}{|c|}{ Postoperative renal function indicators } \\
\hline Group & & $\begin{array}{l}\text { Serum creatinine } \\
\qquad(\mu \mathrm{moL} / \mathrm{L})\end{array}$ & $\begin{array}{c}\text { BUN } \\
(\mathrm{mmoL} / \mathrm{L})\end{array}$ & $\begin{array}{l}\text { Creatinine clearance } \\
(\mathrm{mL} / \mathrm{min})\end{array}$ \\
\hline Control & Postoperative day 1 & $107.0 \pm 15.5$ & $8.4 \pm 2.6$ & $70.2 \pm 24.9$ \\
\hline Control & Postoperative day 2 & $113.7 \pm 15.3$ & $8.5 \pm 2.4$ & $69.0 \pm 22.2$ \\
\hline Control & Postoperative day 3 & $123.6 \pm 17.4$ & $9.4 \pm 2.3$ & $60.6 \pm 23.3$ \\
\hline Observation & Postoperative day 1 & $105.9 \pm 15.9$ & $7.8 \pm 2.5$ & $71.8 \pm 23.8$ \\
\hline Observation & Postoperative day 2 & $112.5 \pm 15.2$ & $8.0 \pm 2.4$ & $70.1 \pm 24.1$ \\
\hline Observation & Postoperative day 3 & $114.6 \pm 17.0^{a}$ & $8.5 \pm 2.1^{a}$ & $69.7 \pm 22.6^{a}$ \\
\hline
\end{tabular}

Data are mean \pm SD; Compared with control group ap $<0.05$ 
2015). When a large number of kidney units were damaged, the plasma level of serum creatinine is significantly increased. CIN prolonged the length of hospital stay, which in turn increased the patients hospital mortality, and in line with the presence of long -term adverse events in patients (Zhao et al., 2015). Therefore, it is necessary to take measures to prevent the occurrence of CIN and reduces its incidence. The current prevention means of CIN include the cessation of nephrotoxic drugs (aminoglycoside antibiotics, nonsteroidal anti-inflammatory drugs, amphotericin B, high-dose loop diuretics, etc.), hydration therapy, rational use of contrast agents (including type and dose of contrast agent) (Rundback et al., 2011). In addition, the use of prophylactic drugs in preoperative (rosuvastatin and other statins, vitamin C, calcium channel blockers, adenosine antagonists, theophylline and prostate E1, etc.), hemodialysis and hemofiltration. Among them, the use of 0.9 or $0.45 \%$ saline for hydration therapy is currently widely recognized for the prevention of CIN (Khanal et al., 2005).

Soma et al. (2002) demonstrated that the incidence of CIN may also be related with the prostate, nitric oxide and other renal vasodilator activity decreased. The treatment of kidney disease is individual differences in Chinese medicine, and mainly improved blood as the center. Furthermore, it focused on the body as a whole conditioning, to help righting to improve the effectiveness of the body immunity. While reducing the side effects of Western medicine through the relevant metabolic pathway and reduce the amount of medicine and other ways (Zhao and Zhang, 2013). Therefore, Chinese medicine has a significant advantage in the treatment of kidney disease, and integrated Chinese and Western medicine treatment program better efficacy. Rhubarb inhibits glomerular mesangial and renal tubular epithelial cell proliferation. Additionally, it reduced the compensatory hypertrophy of damaged kidney and eased the residual renal unit of high metabolic operation. It suggested that rhubarb played an important role in renal disease associated with lipid disorders, and provided essential amino acids and trace elements for body (Jiang et al., 2011). Astragalus has invigorating qi and consolidation of superficies, convergence sweat, subsidies upright and to draft out by sepsis, diuretic swelling effect function. It also improved chronic nephritis and proteinuria diabetes. Danshen prevents small thrombosis in renal and expands renal blood vessels. Safflower has a protective effect in ascending and descending turbidity treatment of chronic renal failure (Fen, 2013). The main components of Shenkang injection were rhubarb, astragalus, salvia and safflower. Many studies have shown that it has obviously reduced turbidity, blood circulation and descending turbid. It suit for chronic renal failure and the recovery of renal function (Jiang et al., 2011). Alprostadil is one of the highly active substances of prostaglandin E1 class. It promotes vasodilation, inhibits platelet aggregation by renal vascular dilation and increases blood flow in kidney. Then it reduced urinary protein excretion and the role of kidney inflammation (Xu et al., 2013). Above all, this study focus on the effect of Shenkang injection combined with alprostadil injection on contrastinduced nephropathyin elderly patients with diabetes mellitus complicated with mild to moderate renal insufficiency.

BUN is an important kidney functional indicator. Plasma BUN levels are affected by major factors such as protein intake, degree of decomposition, and renal blood flow. It indicated that abnormal levels of plasma BUN are present when $60-70 \%$ of renal units were damaged. Clinical serum creatinine concentration is an important indicator of glomerular filtration function. In addition, creatinine clearance also reflects the glomerular filtration function and a rough estimate of the number of effective nephrons.

In this study, there were no significant differences in two groups in the value of serum creatinine, BUN and creatinine clearance in preoperative and postoperative 1 $-2 \mathrm{~d}(\mathrm{p}>0.05)$. After 3 days, the level of serum creatinine, BUN and creatinine clearance were significant difference in two groups $(\mathrm{p}<0.05)$. The results showed that the incidence of CIN in the observation group was significantly lower than that in the control group, and the curative effect of TCM in observation group was significantly improved $(\mathrm{p}<0.05)$. It indicated that the incidence of CIN may be related to the expansion of the prostate and other substances related to the reduction of renal activity. The mechanism of prevention of CIN morbidity may be related to increased vasodilator prostate activity. Cirit et al. (2006) showed that increased albumin may also be one of the risk factors for CIN morbidity. Liao et al. (2015) found that alprostadil decreased urinary albumin in elderly patients with diabetes and chronic kidney disease. Therefore, the mechanism of alprostadil to prevent CIN may also be related to reducing the amount of albumin in the blood. However, the exact mechanism of alprostadilin prevention of CIN has not yet been confirmed, and further studies are needed.

\section{Conclusion}

Shenkang injection combined with alprostadil injection improved the CIN in elderly patients with diabetes mellitus complicated with mild to moderate renal insufficiency.

\section{Ethical Issue}

The trial has been approved by the Ethics Committee of 
the Court and signed with the informed consent by patients and family members.

\section{Conflict of Interest}

The authors have no conflict of interest in this paper.

\section{References}

Cirit M, Toprak O, Yesil M, Bayata S, Postaci N, Pupim L, Esi E. Angiotensin-converting enzyme inhibitors as a risk factor for contrast-induced nephropathy. Nephron Clin Pract. 2006; 104: c20-27.

Feldkamp T, Kribben A. Contrast media induced nephropathy: Definition, incidence, outcome, pathophysiology, risk factors and prevention. Minerva Med. 2008; 99: 177-96.

Fen XZ. Wang systematic mechanism of Rhubarb and Chloostatum on restoring signaling pathway of renal tubular epithelium in rats with contrast-induced nephropathy. Shanghai J Tradit Chin Med. 2013; 47: 37-38.

Gleeson TG, Bulugahapitiya S. Contrast-induced nephropathy. Am J Roentgenol. 2004; 183: 1673-89.

Isobe S, Yamada T, Sato K, Murohara T. Diabetes with preserved renal function is an independent risk factor for renal function deterioration after coronary computed tomography angiography. J Comput Assist Tomogr. 2013; 37: $750-54$.

Jiang ZW, Lv YY, Xia JL. Clinical observation on the treatment of chronic renal failure with Shenkang injection. J Chin Med Univ. 2011; 40: 941-45.

Kato K, Sato N, Yamamoto T, Iwasaki Y-k, Tanaka K, Mizuno K. Valuable markers for contrast-induced nephropathy in patients undergoing cardiac catheterization. Circulation 2008; 72: 1499-505.

Khanal S, Attallah N, Smith DE, Kline-Rogers EM, Share DA, O'Donnell MJ, Moscucci M. Statin therapy reduces contrastinduced nephropathy: An analysis of contemporary percutaneous interventions. Am J Med. 2005; 118: 843-49.

Li WM, Xue JY. Comparison of the progress of contrastinduced nephropathy. Chin J Circ. 2010; 25: 325-26.

Li W, Zhan F, Wu W. Effect of alprostadil on patients with acute kidney injury. Chin Trop Med. 2012; 11: 42.
Liao SJ, Yang C, Jiang HQ. Effect and safety of alprostadil in the treatment of elderly patients with diabetes mellitus and chronic kidney disease. J Zhongshan Univ. 2015; 36: 120-25.

Lu ZY, Cheng DS, Yin JY, Wu R, Zhang GY, Zhao Q, Wang NS, Wang F, Liang MY. Antithrombin III protects against contrast-induced nephropathy. EBioMedicine 2017; 17: 10107 .

Lv RH, Zhang FR, Gao YB. Chinese medicine stage syndrome evaluation criteria of diabetes. Chinese medicine diagnosis treatment of diabetes. 1999, pp 363-65.

Menting TP, Sterenborg TB, DeWaal Y, Donders R, Wever KE, Lemson MS, van der Vliet JA, Wetzels JF, Schultzekool LJ, Warle MC. Remote ischemic preconditioning to reduce contrast-induced nephropathy: A randomized controlled trial. Eur J Vasc Endovasc Surg. 2015; 50: 527-32.

Miao Y, Zhong Y, Yan H, Li W, Wang BY, Jin J. Alprostadil plays a protective role in contrast-induced nephropathy in the elderly. Int Urol Nephrol. 2013; 45: 1179-85.

Parfrey PS, Griffiths SM, Barrett BJ, Paul MD, Genge M, Withers J, Farid N, McManamon PJ. Contrast materialinduced renal failure in patients with diabetes mellitus, renal insufficiency, or both. N Engl J Med. 1989; 320: 143-53.

Rundback JH, Nahl D, Yoo V. Contrast-induced nephropathy. J Vasc Surg. 2011; 54: 575-79.

Soma VR, Cavusoglu E, Vidhun R, Frishman WH, Sharma SK. Contrast associated nephropathy. Heart Dis. 2002; 4: 372-79.

Wang XD, Shen JP. Experimental study on the effect of Shenkang injection on prevention of contrast-induced nephropathy after aged coronary artery disease. Nanjing Univ Tradit Chin Med. 2013; 29: 16-17.

Xu RH, Ma GZ, Cai ZX, Chen P, Zhu ZD, Wang WL. Combined use of hydration and alprostadil for preventing contrast-induced nephropathy following percutaneous coronary intervention in elderly patients. Exp Ther Med. 2013; 6: 863-67.

Zhao B, Zhao Q, Li J, Xing T, Wang F, Wang N. Renalase protects against contrast-induced nephropathy in SpragueDawley rats. PLoS One. 2015; 10: e0116583.

Zhao K, Li Y, Zhang H. Role of Dongchongxiacao (Cordy-ceps) in prevention of contrast-induced nephropathy in patients with stable angina pectoris. J Tradit Chin Med. 2013; 33: 28386. 